\title{
Two-dimensional flow visualization and velocity measurement in natural convection near indoor heated surfaces using a thermal image velocimetry method
}

Article

Accepted Version

Creative Commons: Attribution-Noncommercial-No Derivative Works 4.0

Wu, Q., Zhu, C.-a., Liu, L., Liu, J. and Luo, Z. (2019) Twodimensional flow visualization and velocity measurement in natural convection near indoor heated surfaces using a thermal image velocimetry method. Applied Thermal Engineering, 146. pp. 556-568. ISSN 1359-4311 doi: https://doi.org/10.1016/j.applthermaleng.2018.10.023 Available at https://centaur.reading.ac.uk/79752/

It is advisable to refer to the publisher's version if you intend to cite from the work. See Guidance on citing.

To link to this article DOI: http://dx.doi.org/10.1016/j.applthermaleng.2018.10.023

Publisher: Elsevier

All outputs in CentAUR are protected by Intellectual Property Rights law, including copyright law. Copyright and IPR is retained by the creators or other copyright holders. Terms and conditions for use of this material are defined in the End User Agreement. 


\section{www.reading.ac.uk/centaur}

\section{CentAUR}

Central Archive at the University of Reading

Reading's research outputs online 


\title{
Two-dimensional flow visualization and velocity measurement in natural convection near indoor heated surfaces using a thermal image velocimetry method
}

\author{
Qing Wu ${ }^{\text {a }}$, Chang-an $\mathrm{Zhu}^{\mathrm{c}}{ }^{\mathrm{c}}$, Lin Liu ${ }^{\mathrm{a}}$, Jing Liu ${ }^{\mathrm{a}, \mathrm{b}, *}$, Zhiwen Luo ${ }^{\mathrm{d}, *}$ \\ ${ }^{a}$ School of Architecture, Harbin Institute of Technology, Harbin 150000, China. \\ ${ }^{b}$ Heilongjiang Cold Region Architecture Science Key laboratory, Harbin 150000, China \\ ${ }^{c}$ China Southwest Architecture Design and Research Institute Co., Ltd, Chengdu 610000, China. \\ ${ }^{d}$ School of the Built Environment, University of Reading, UK.
}

\begin{abstract}
Indoor velocity measurement techniques are categorized into point-wise and globalwise measurement techniques. Currently, measurements are either intrusive or restricted to the measurement area. This study presents a thermal image velocimetry (TIV)-based flow measurement method that is suitable for visualizing indoor two-dimensional velocity fields near indoor heated surfaces. The proposed technique uses only an infrared camera for mapping the surface temperature fluctuations. Image processing steps that are used to recover the velocity distribution include the decomposition of the video files into individual frames, the application of filtering to remove background noise, cross-calculation to estimate the velocity, and a final velocity correction based on the continuity equation. To investigate the feasibility of this method, natural convection was studied close to a heated vertical surface in a rectangular cavity. Thermal image velocimetry and particle image velocimetry (PIV) were used to visualize the flow field above a heating unit. The results indicate that the airflow field can be visualized by TIV, and the results measured by TIV are shown to be similar to those for the surface of $6 \mathrm{~mm}$ away from the heated surface measured by PIV. A linear correlation is established between TIV and PIV.
\end{abstract}

Keywords: Thermal image velocimetry; Heated surface; Particle image velocimetry; Indoor airflow.

*Corresponding author: Jing Liu, Zhiwen Luo

Address: School of Architecture, Harbin Institute of Technology, No.73, Huanghe Road, Nangang District, Harbin 150000, China (J. Liu).

Tel./fax: +86045186282123.

E-mail: liujinghit0@163.com(J. Liu), z.luo@,reading.ac.uk (Z. Luo). 


\section{Abbreviations}

TIV thermal image velocimetry

PIV particle image velocimetry

30

Symbols

$y$

$t_{1}$

$t_{2}$

$u$

$v$

\section{V}

val

$r$

$N \quad$ the number of the vectors in the entire flow field

$n \quad$ the query value to find the critical grid when the val values of all the grids are arranged in ascending order

valn the val value of the critical grid

$u_{n} \quad$ the horizontal velocity of the critical grid for velocity correction $(\mathrm{m} / \mathrm{s})$

$v_{n} \quad$ the vertical velocity of the critical grid for velocity correction $(\mathrm{m} / \mathrm{s})$

$v_{\max }$ the maximum velocity along a horizontal line near the warm vertical surface $(\mathrm{m} / \mathrm{s})$

$H \quad$ the distance from the lower level of the heated vertical surface (m)

$T_{o} \quad$ target surface temperature $\left(\mathrm{T}\right.$ or $\left.{ }^{\circ} \mathrm{C}\right)$

$T_{u} \quad$ atmospheric temperature $\left(\mathrm{T}\right.$ or $\left.{ }^{\circ} \mathrm{C}\right)$

$T_{a} \quad$ ambient air temperature $\left(\mathrm{T}\right.$ or $\left.{ }^{\circ} \mathrm{C}\right)$

$K \quad$ sampling size (pairs)

$z_{\alpha / 2} \quad$ coefficient determined by the confidence interval

$a^{\prime} \quad$ root mean square velocity $(\mathrm{m} / \mathrm{s})$

$A$ time-averaged velocity $(\mathrm{m} / \mathrm{s})$

$S(A) \quad$ the random sampling error of the time-averaged velocity (\%)

$E_{b \lambda} \quad$ the black body radiation intensity $\left(\mathrm{W} / \mathrm{m}^{2}\right)$

$E_{\lambda} \quad$ the radiant intensity detected by thermal infrared camera $\left(\mathrm{W} / \mathrm{m}^{2}\right)$

PIV6 PIV measurement results of the surface of $6 \mathrm{~mm}$ away from the heated surface

PIV12 PIV measurement results of the surface of $12 \mathrm{~mm}$ away from the heated surface

31 Greek letters

$\alpha \quad$ horizontal displacement of the thermal spot (m)

$\beta \quad$ vertical displacement of the thermal spot (m)

$\Delta \theta \quad$ the temperature difference between the wall and the adjacent air ( $\mathrm{T}$ or ${ }^{\circ} \mathrm{C}$ )

$\varepsilon_{\lambda} \quad$ target surface emissivity

$\tau_{a \lambda} \quad$ atmospheric spectral transmittance

32 Subscripts

the horizontal sequence numbers of the nine grids consisting $-1,0,1$ 


\section{Introduction}

Accurate quantification of airflow fields is of great significance in evaluating and creating healthy indoor environments [1]. The currently conducted research on airflow fields can be implemented using experiments and numerical simulations. Typically, numerical simulation relies on accurate boundary conditions and experimental validation [1]. By contrast, experiments can provide primary raw data of the actual airflow field and, therefore, is more reliable and fundamental. Traditionally, experimental methods can be classified into two primary categories: point-wise and global-wise measurements [1]. The point-wise technique obtains velocity information at predetermined representative points by arrangement of velocity measurement probes. Hot-wire anemometer, hot-sphere anemometer, ultrasonic anemometer, and laser Doppler velocimetry are common types of point-wise measurement techniques. Typically, point-wise measuring systems are relatively precise and easy to operate. In addition, anemometers have fast response times and are sufficient for turbulent measurements. Therefore, this method is widely used in numerous indoor airflow measurement systems [2]-[5]. Global-wise measurement, such as particle image velocimetry (PIV), particle tracking velocimetry, and particle streak velocimetry, can estimate the twodimensional or three-dimensional velocity vector distributions of an entire domain, and are applied in numerous types of indoor airflow measurement systems [6]-[9]. Of these techniques, PIV is thought to be the most extensively used optical velocimetry method for indoor airflow measurements given its mature development level, an abundance of experimental literature, and the availability of commercial systems [10]. Compared to point-wise measurements, the ability to obtain detailed flow pattern makes PIV more advantageous for measuring extensive airflow fields.

The airflow measurement close to a heated surface is an important aspect for indoor airflow measurements. Dominated by buoyancy originating close to a heated surface, the airflow is significantly complex, and a thorough understanding of its characteristics is of great significance for studies on room thermal comfort and energy efficiency [11]. The rising airflow induced by 
buoyancy generated close to the heated wall contributes to the air circulation in an entire room, and

can alleviate the heat loss and the thermal discomfort induced by the infiltration of cold air. To date, there have been limited experimental studies [11]-[14] investigating the airflow field close to heated surfaces. This discrepancy can be attributed to the complexities of the building environment and the limitations of measurement technologies. The abovementioned measurement technologies have rarely been successfully applied in actual measurements close to heated surfaces. The primary reasons for this regarding point-wise measurements can be summarized as follows: a) because of the complexity of airflow near the heated surface, it is difficult to predetermine representative points indicative of the characteristics of the entire airflow field, b) the bulk of the point-wise probes are relatively bulky and the arrangement of probe arrays would disturb the flow field and significantly influence the accuracy of the results, and c) for probes that are electrically heated, such as hot-film anemometers, additional thermal buoyancy is experienced around them, and this would introduce considerable errors into the results [1]. For global-wise measurements, there are also practical difficulties in the application of measurements close to heated surfaces. Specifically, a) tracer particles accumulate readily on an actual surface that has a certain degree of roughness, causing the particle concentration to be uneven in space, ultimately affecting the measurement, and b) because of the limitations of the optical path and camera resolution, it is rarely applied in full-scale measurements and the area is typically smaller than $1 \mathrm{~m}^{2}[15]$. and has been used for years in a variety of fields, including geography, military, medicine, and aviation [16]-[19]. Compared to standard techniques, such as thermocouples, the thermal camera 
on this property, an infrared camera can be used to estimate the temperature and compute the heat flux of complex flows [20] [21] [22]. Because of the high-spatial resolution and the ability to sample the spatial and temporal changes of surface temperatures, infrared thermography has significant potential in climatological studies. A. Christen et al. [23] and R. Voot et al. [24] observed significant high-frequency fluctuations on the street canyon using infrared thermography and found that the fluctuations were controlled by near-surface winds. They confirmed that there was a link between surface temperature fluctuations and adjacent turbulent air movements. Based on the resolution of surface temperature fluctuations caused by coherent heat exchanging between land surfaces and the atmosphere using thermal imagery, additional studies have been conducted [25]-[30]. However, these studies cannot provide the velocity field directly and merely infer the turbulent state according to the temperature fluctuations. In current research study, thermal infrared velocimetry (TIV) based on time-sequential infrared thermography [31] was used for outdoor airflow analysis. Based on this theory, Y. Fan et al. [32] and A. Tiddens et al. [33] investigated the flow fields close to outdoor walls and the river flow respectively. In spite of the considerable potential of thermography, its utilization in flow visualization is still at an early stage of development. In current studies, only qualitative vector fields can be provided, and studies of the indoor airflow measurement using TIV are limited.

In order to analyze the application of TIV in measuring indoor two-dimensional flow fields close to indoor heated surfaces, a scaled experimental cavity was set up and the natural convection velocity field near the heated surface was studied. The aims of this study are as follows: (a) propose a TIV-based method suitable for indoor airflow measurements near the heated surfaces, (b) visualize the near-wall airflow field by TIV and PIV, and (c) compare the data obtained from these two 
methods, analyzing the feasibility of the TIV in the measurement of the near-wall airflow fields.

\section{TIV-based velocity measurement method near heated surfaces}

\subsection{Theory background of TIV}

The infrared detectors detect the infrared radiant energy of the target surface, and the radiant energy is then converted to electrical signals in the signal processing system and processed into the thermal image [34]. In the actual measurement, numerous factors, such as target surface emissivity, target surface reflectivity, background radiation, atmospheric attenuation, measurement distance, and ambient air temperature, affect the measurement accuracy [35]. The radiation received by the thermal camera primarily includes the target radiation, ambient reflection, and atmospheric radiation, that are described below.

$$
E_{\lambda}=\tau_{a \lambda} \varepsilon_{\lambda} E_{b \lambda}\left(T_{o}\right)+\tau_{a \lambda}\left(1-\varepsilon_{\lambda}\right) E_{b \lambda}\left(T_{u}\right)+\left(1-\tau_{a \lambda}\right) E_{b \lambda}\left(T_{a}\right)
$$

where $E_{\lambda}$ is the radiation intensity detected by the thermal infrared camera and $E_{b \lambda}$ is the black body radiation intensity, and $T_{o}, T_{u}, T_{a}$ are the target surface temperature, atmospheric temperature, and ambient air temperature, respectively, $\varepsilon_{\lambda}$ is the target surface emissivity, $\tau_{a \lambda}$ is the atmospheric spectral transmittance.

The energy detected by the thermal camera depends on both the surface emissivity coefficient and the environmental conditions. In Eq. (1), the term $\left(1-\tau_{a \lambda}\right) E_{b \lambda}\left(T_{a}\right)$ is the emission from the atmosphere and $\tau_{a \lambda}$ can be assumed to be equal to 1 when the camera is positioned close to the target surface in indoor measurements. Correspondingly, $\tau_{a \lambda}\left(1-\varepsilon_{\lambda}\right) E_{b \lambda}\left(T_{u}\right)$ is the reflected emission from the surrounding surfaces and $\tau_{a \lambda} \varepsilon_{\lambda} E_{b \lambda}\left(T_{o}\right)$ is the emission from the measured surface captured by the camera. From Eq. (1), it can be concluded that the surface emissivity for a wavelength detectable by the thermal camera should be sufficiently great to decrease the background noise signals. In addition, when the target surface temperature is close to the background temperature, the noise would significantly influence the accuracy of the measurement. Therefore, the temperature difference between the surface and adjacent air should be adequate. Regarding the airflow near the heated surface, a relatively great temperature difference can be 
guaranteed between the heated surface and the adjacent air. In addition, the condition of the wall is also conducive to TIV. The surface of a wall is relatively rough with a small albedo and high emissivity. The significant surface emissivity increases the radiation detectable by the thermal infrared camera and decreases the interference of ambient surfaces.

The link between the surface temperature and energy balance is introduced to study the relationship between temperature and velocity [31]. Energy transfer comprises four parts: heat storage, heat conduction, turbulent heat exchange, and radiation energy transfer. These four parts have a joint effect on the surface temperature and adjacent air temperature. For indoor environments, the turbulent heat exchange plays the leading role in the interaction between the wall surface and air. Driven by buoyancy, convection flow is formed near the heated surface and influences the turbulent heat transfer. Because of the development of turbulent structures, the heat transfer between the wall surface and adjacent air is promoted or suppressed. The trace of turbulent structures will leave a "footprint" on the surface temperature distribution, and this "footprint" changes with the velocity near the heated surface [31]. Based on the temporal variation of the near wall temperature distribution measured by infrared camera, a cross-correlation analysis similar to PIV is performed to calculate the flow velocity. In the next section, the processing steps of TIV are introduced in detail.

\subsection{Data processing for TIV}

Similar to the data processing of PIV, TIV measures the two-dimensional velocity by evaluating the displacement of thermal spots between two temporally continuous images. The technical approach used in this study comprises four steps, as shown in Fig. 1.

Fig. 1. Technical approach

\subsubsection{Step 1: Preparing thermal images}

Data collection involves the acquisition of a temperature fluctuation video of the target object by means of an infrared camera. The ResearchIR software is provided by FILR for research and science cameras and can be used for camera control and high-speed data recording. In this step, we use the ResearchIR software to specify the frame rate and the duration of each image time series. First, the raw .ats video files are obtained by the infrared camera. These files are then imported into 
MATLAB and are parsed into image stacks comprising individual frames.

\subsubsection{Step 2: Pre-processing}

During the experiment, there is a need to accentuate fluctuations of the surface temperature compared to the background. In the case of TIV technology, the local heterogeneities of the surface also influence the surface temperature pattern [31]. The image enhancement aims at reducing the influence of background noise [36]. A filter serves as a frequency selection tool and allows specific frequency signals to pass and blocks other frequency signals. Proper filtering is critical in the process of image enhancement. As the storage of digital images is discrete, the discrete Fourier transform is commonly used [37]. Typically, through the use of the fast Fourier transform the spatial domain $f$ $(\mathrm{x}, \mathrm{y})$ is converted to a frequency domain, and the frequency domain is then multiplied by the filter transfer function. The filtered results are then obtained by the inverse fast Fourier transform. Through optimization in numerous preliminary experiments, the Gaussian high-pass filter is used in this study.

\subsubsection{Step 3: Image processing}

Similar to PIV, TIV estimates the velocity according to cross-correlation calculation [38]. Fig. 2. shows the data processing procedure for TIV measurement.

Fig. 2. The data processing for TIV measurement

The images are divided into small calculation sections that are similar to the interrogation windows in the PIV algorithm. As shown in Fig. 3, the thermal spot is located in one position $(x, y)$ in the first image aat time $t_{l}$ and in $(x+\alpha, y+\beta)$ at time $t_{2}$ for the second image. The crosscorrelation calculation is executed within the calculation section to identify the point with maximum correlation. The calculation section is of $64 \times 64$ image pixels, $50 \%$ overlapped. Through the identification of the maximum correlated point, the displacement of the thermal spot is identified. The $x$-component of velocity $(u)$ and the $y$-component of velocity $(v)$ are calculated according to the displacement of the point divided by the time increment between the two images. The calculation section is shifted subsequently in the image to evaluate the entire flow field. 

flow field is not considered. The output from step 3 that is based on the cross-correlation method comprises a number of vectors that cannot satisfy the fundamental law of fluid dynamics. They are either appreciably different from neighboring vectors or outside the physically possible velocity range [39]. In this process, val is defined as the value descripting the uniformity degree of the grids and the raw vectors can be validated by analyzing the val of each grid that is calculated using the eight closest neighboring vectors, as shown as Fig. 3. The location where val is sufficiently small indicates that the vector in this grid reaches a degree of uniformity. The val is defined according to the continuity equation [40]. For incompressible fluid, the two-dimensional fluid continuity equation is:

$$
\frac{\partial u}{\partial x}+\frac{\partial v}{\partial y}=0
$$

Fig. 3. The sketch for velocity grid in the step of data correction

In the two-dimensional vector field, the correct velocity vector should follow the continuity equation. The two-dimensional fluid continuity equation at the grid position $(0,0)$ can be described:

$$
\begin{gathered}
\frac{\partial u}{\partial x}+\frac{\partial v}{\partial y}=\sum_{i, j}\left[\left(u_{i, j}-u_{0,0}\right) \operatorname{sgn}(i)+\left(v_{i, j}-v_{0,0}\right) \operatorname{sgn}(j)\right]=0 \\
\operatorname{sgn}(\delta)=\left\{\begin{array}{cc}
1, & \delta \geq 0 \\
-1, & \delta<0
\end{array}\right.
\end{gathered}
$$

where $u_{0,0}$ and $v_{0,0}$ are the horizontal and vertical velocities at the grid position $(0,0)$ respectively; $u_{i, j}$ and $v_{i, j}$ indicate the horizontal and vertical velocities of the eight grid positions around grid location $(0,0)$. According to Eq. (3), the following inequalities can be derived, as shown as Eq. (5) and Eq. (6). The val at the grid location $(0,0)$ in the two-dimensional velocity vector field can be calculated based on Eq. (7).

$$
\begin{gathered}
\frac{\partial u}{\partial x}+\frac{\partial v}{\partial y} \leq \sum_{i, j}\left|u_{i, j}\right|+\sum_{i, j}\left|v_{i, j}\right| \\
\frac{\partial u}{\partial x}+\frac{\partial v}{\partial y} \leq \sum_{i, j}\left|u_{i, j}-u_{0,0}\right|+\sum_{i, j}\left|v_{i, j}-v_{0,0}\right|
\end{gathered}
$$




$$
v a l=\frac{\sum_{i, j}\left|u_{i, j}-u_{0,0}\right|+\sum_{i, j}\left|v_{i, j}-v_{0,0}\right|}{\sum_{i, j}\left|u_{i, j}\right|+\sum_{i, j}\left|v_{i, j}\right|}
$$

201

In this way, the val of each grid point in the two-dimensional velocity vector field can be calculated and then they are arranged in ascending order. Two parameters $r$ and $N$, that are the percentage of the vectors that are different from neighboring vectors or outside the physically possible velocity range and the number of vectors in the entire flow field, respectively, should be defined in the described algorithm. In this study, $r$ was set to $6 \%$, and $N$ was multiplied by $r$ to specify the value of the query values $n$. According to $n$, the corresponding valn in the ascending array can be identified, and the corresponding grid location and the $x$ and $y$-components of the velocities $u_{n}$ and $v_{n}$ can be obtained. By comparison with $u_{n}\left(v_{n}\right)$, the velocities in the entire velocity field that are smaller than the critical values $u_{n}$ and $v_{n}$ are corrected using Eq. (8). In this case, by using the abovementioned algorithm, the entire flow field is corrected based on the physical properties of the fluid.

$$
u_{0,0}=\frac{\sum_{i, j} u_{i, j}}{8} \quad v_{0,0}=\frac{\sum_{i, j} v_{i, j}}{8}
$$

\section{Scaled model experiment}

\subsection{Experimental cavity}

Experiments were conducted in a closed rectangular cavity with internal dimensions $1200 \times$ $500 \times 1400 \mathrm{~mm}^{3}$, as shown in Fig. 4. The walls were made of 8-mm-thick Plexi-glass with a surface emissivity of 0.90 . The cavity was built in an air-conditioned room with the room temperature set to $20{ }^{\circ} \mathrm{C}$. An electric heating unit with dimensions $500 \times 200 \mathrm{~mm}^{2}$ was mounted on the bottom of one of the vertical walls. A 5-mm-thick foam sheet with a thermal conductivity of $0.2 \mathrm{~W} /(\mathrm{m} \cdot \mathrm{K})$ was placed between the heating unit and the heated vertical wall. The foam sheet was used as an insulation material to reduce the heat loss through the rear of the heated vertical wall. In order to maintain the surface temperature, the heating unit was controlled by an electronic thermostat. In this study, the surface temperature of the heating unit was determined to be $50^{\circ} \mathrm{C}$.

Fig. 4. Experimental setup: 1. Tracer particle inlet 2. Heating unit 3. CCD camera 4. Laser 5. Infrared camera 6. Visualized surface 


\subsection{Measurement system and domain composition}

\subsubsection{PIV measurement system and domain composition}

The PIV system comprised a 15-Hz Nano L PIV, 135 mJ, 532 nm, double-pulsed Nd:YAG laser used as a light source, in association with a double-frame, high sensitivity CCD camera PIVCAM13-8 with a resolution of $2048 \times 2048$ pixels. An AF Nikko $50 \mathrm{~mm} \mathrm{f} / 1.8 \mathrm{D}$ objective lens was mounted on the CCD camera. The thickness of the laser light sheet at the measurement plane was approximately 1-2 mm. In order to capture the actual airflow motion, tracer particles with proper intensity and size are required. A VZ09 - 0751 smoke generator was used, and was connected to the plenum located on the bottom of the cavity. The tracer particles were released into the cavity from the inlet.

If the CCD camera is positioned on the same side as the infrared camera, the pixels occupied by the particles are significantly decreased, even though the shooting area increases. In addition, the tracer particles suspended in the cavity significantly influence the quality of the measurement. As a result, the CCD camera was positioned on the opposite side. Because of the existence of the heating unit and the air plenum in the upper part of the heated vertical wall, the visualized area for PIV was $500 \times 1000 \mathrm{~mm}^{2}$, as shown in Fig. 5 . By adjusting the position of the CCD camera in the preliminary experiment, the dimensions of the image obtained by the CCD camera were determined to be 280 $\mathrm{mm} \times 280 \mathrm{~mm}$. The regions were set to have horizontal and vertical overlaps of $60 \mathrm{~mm}$ and $30 \mathrm{~mm}$, respectively, with the neighboring domains shown in Fig. 5(a). For each domain measurement, 300 PIV image pairs were collected at a recording frequency of $7 \mathrm{~Hz}$. The velocity vector maps were prepared by determining the cross-correlation for $64 \times 64$ pixel interrogation areas and $50 \%$ overlaps in both the $x$ and $y$ directions for all PIV image pairs. For each domain, the instantaneous velocities for 300 image pairs and an average velocity field were obtained. The statistical data associated with eight regions were exported to MATLAB for connecting the eight flow fields.

Fig. 5. Schematic of measurement domains a. PIV b. TIV

\subsubsection{TIV system and domain composition}

A FLIR-T1040 infrared camera was used to track the flow of the surface near the heated wall. 
The camera has a resolution of $1024 \times 768$ pixels and is sensitive in a range $7.5-14 \mu \mathrm{m}$, thereby being suitable for indoor temperature measurements. In addition, it is able to resolve subtle differences in indoor temperature (noise-equivalent temperature difference $<25 \mathrm{mK}$ ). A telephoto lens with a focal length of $25 \mathrm{~mm}$ was mounted on the camera to provide a data field of view of $28^{\circ}$ $\times 21^{\circ}$. As the wavelength of the PIV laser is $532 \mathrm{~nm}$ and the sensitive wavelength range of the infrared camera is $7.5-14 \mu \mathrm{m}$, the PIV and TIV measurements were performed separately. As shown in Fig. 4, there are three shooting positions located $250 \mathrm{~mm}, 600 \mathrm{~mm}$, and $950 \mathrm{~mm}$ from the bottom of the cavity and three ats video files were recorded from the three shooting positions. Based on the horizontal distance between the shooting positions and the visualized surfaces, the imaged area for one time is $598 \times 445 \mathrm{~mm}^{2}$. The entire area that was investigated was decomposed into three domains with an overlap of $68 \mathrm{~mm}$, as shown in Fig.5 (b). The sampling frequency was $10 \mathrm{~Hz}$ and the fluctuations of the temperature field were captured by the thermal infrared camera. Four hundred instantaneous velocities at a frame rate of $10 \mathrm{~Hz}$ were extracted to calculate the average velocity. Finally, the flow field measured by TIV was mirrored to compare with the PIV results.

\section{Results}

\subsection{The flow characteristics of heat plume}

Fig. 6 shows the changing tendency of the heat plume in consecutive moments. It indicates that the primary energy transmission direction is upward. A hot plume is first generated over the heating unit and a significant velocity gradient is generated adjacent to the heating unit. The heated air moves upward, and the space left by the rising hot plume is supplemented by cold air in the upper part of the cavity. The two airflow structures mix and momentum and energy are exchanged. The velocity gradient changes at a relatively lower rate and the entire flow field tends to be more uniform.

Fig. 6. Instantaneous velocity measured by TIV, with a to $\mathbf{d}$ representing four consecutive moments. According to a relevant investigation by [11], the air velocity induced by the convection near a warm or cold vertical surface can be manipulated as follows:

$$
v_{\text {max }}=0.1 \sqrt{\Delta \theta H}
$$


the temperature difference between the wall and the adjacent air, and $H$ is the distance from the lower level of the heated vertical surface. As debated by [11], Eq. (9) can provide an estimation of the maximum velocity adjacent to the heated surface, but not above it. It is also assumed that this equation is suitable for the region above the heating surface, that is not at a great distance from the upper edge of the heating surface. In this study, the temperature difference between the heated surface and air is approximately $30^{\circ} \mathrm{C}$ according to the temperature difference of the heating unit and the room temperature. At a height of $200 \mathrm{~mm}$, that is $400 \mathrm{~mm}$ above the bottom of the heating unit, the maximum velocity is $0.28 \mathrm{~m} / \mathrm{s}$, as estimated by Eq. (9). The result obtained by TIV is $0.26 \mathrm{~m} / \mathrm{s}$, that is similar to the result of Eq. (9).

\subsection{Mean flow fields measured by TIV and PIV}

The surfaces at $6 \mathrm{~mm}$ and $12 \mathrm{~mm}$ away from the heated vertical surface were visualized using PIV technology. In the next sections, the PIV results of surfaces at $6 \mathrm{~mm}$ and $12 \mathrm{~mm}$ away from the heated surface are referred to as PIV6 and PIV12, respectively. The instantaneous flow fields are averaged, and the averaged velocity contour and vectors measured by PIV and TIV of the region over the heating unit are shown in Fig. 7 and Fig. 8, respectively. All measurements were conducted under steady-state conditions attained after a sufficiently long period. The infrared camera was used to monitor the temperature variations of the near wall surface over the heating unit and the steadystate was deemed to be reached when the temperature remained approximately constant. From a visual inspection of Fig. 7 (a) and (c), and Fig. 8 (a), the spatial variability of the velocity appears to comprise three patterns from the bottom to the top: (1) a high velocity region, (2) a moderate velocity region, and (3) a low velocity and lagging region. Pattern 1 is attributed to the intense energy and momentum exchange between the air and the heating unit. It is clear that the air adjacent to the heating unit is heated and this causes the velocity to increase significantly. Over this region is pattern 2, where, the airflow part rises in the original direction, and the other part could move to the inner regions of the cavity because of an insufficient driving force. The transition point between patterns 1 and 2 appears at a vertical height of $200 \mathrm{~mm}$ for the surface that is $6 \mathrm{~mm}$ away from the heated surface, and at $400 \mathrm{~mm}$ for the surface that is $12 \mathrm{~mm}$ away from the heated surface, as shown as Fig. 7 (a) and (c). In pattern 3, the velocity decreases to a minimum and vortices appear in the 
corners close to the sidewalls, as shown as Fig. 8 (b) and Fig. 7 (b) and (d). The primary air motion is upward, and the flow direction changes close to the top of the wall.

Overall, the TIV results are in good agreement with the PIV6 results shown in Fig. 7 (a) and Fig. 8 (a). However, there is still some differences in the upper and lower regions. In the middle of the lower region located at $y=0-200 \mathrm{~mm}$, the velocity evaluated by TIV is smaller than the PIV results. In this region, there exists greater heat and momentum transfer compared to the other regions. In the lower region, the TIV measurement is more susceptible to the influence of shadows on the glass target surface and thus affect the tracking of temperature fluctuations. Consequently, the measured temperature fluctuations are smaller in the middle region and the evaluated velocity is accordingly smaller. With regard to the upper regions located at $y=800-1000 \mathrm{~mm}$, the velocity measured by TIV is also smaller than that of PIV. As stated above, for the application of the TIV method, a relatively significant temperature difference is required. In the upper region of the measurement domain, the temperature difference is smaller, and this influences the quality of the results. The velocity evaluated by TIV is relatively smaller than the PIV results for this reason.

Fig. 7. Velocity distributions near the heated surface evaluated by PIV: a (b), c (d) velocity contour (velocity vector) of the planes $6 \mathrm{~mm}$ and $12 \mathrm{~mm}$ away from the heated surface.

Fig. 8. Velocity distributions near the heated surface evaluated by TIV: a velocity contour, b velocity vector.

The histogram of the $y$-component of the velocity $(v)$ at $(200,200)$ obtained by TIV and PIV6 is plotted in Fig. 9. Note that the $x$-component of the velocity is extremely small in magnitude and is not plotted. The overall shapes of the distributions are similar. It is noteworthy that both frequencies are approximately normally distributed. The measured $y$-component of the velocity $(v)$ is characterized by a relative broad spectrum with a maximum of $0.19 \mathrm{~m} / \mathrm{s}$ for both PIV6 and TIV. There is a good agreement between PIV6 and PIV, therefore, the statistical values can be taken as reliable. It is interesting to notice that $0.19 \mathrm{~m} / \mathrm{s}$ is a critical value. In the interval of $0.1-0.19 \mathrm{~m} / \mathrm{s}$, the measured frequency of PIV6 is greater than that of TIV. However, the rule is modified in the interval of $0.20-0.30 \mathrm{~m} / \mathrm{s}$. From Fig. 9, it can be deduced that the TIV measurements could be appreciably greater than those of PIV6 and there appeared to be a correlation between them.

Fig.9. Histogram of $y$-component of the velocity $(v)$ evaluated by TIV and PIV6. 


\subsection{Analysis of velocity in representative lines}

A further comparison and analysis of the results of TIV and PIV is performed. Three vertical lines at locations $x=125 \mathrm{~mm}, 250 \mathrm{~mm}$, and $375 \mathrm{~mm}$, as shown in Fig. 10(d), were chosen as representative lines, and the $y$-components of the velocities of these lines are analyzed. The three lines are referred to as Lines 1,2 , and 3 . The $y$-component of the velocity $(v)$ along the three vertical lines at different locations along the $x$-axis are shown in Fig. 10. Overall, the $y$-components of the velocities exhibited a distinct trend, that is marked by an increase toward a maximum value close to $y=0-200 \mathrm{~mm}$, and by a subsequent decrease to a minimum value at the top of the cavity. Variations were observed in the $y$-component of the velocity close to $y=200 \mathrm{~mm}$. In this region, the heat gradient is developed and warm fluid adjacent to the heating unit is transmitted upward, and is then replaced by the upward cold air. This is a region that is adjacent to the heated surface, and where a velocity $y$-component peak exists. In addition to the decreasing buoyancy, the change in the flow direction could also elicit a sudden decrease close to $y=200 \mathrm{~mm}$. Movement toward the inner parts of the cavity could exist, and the increase in the velocity normal to the heated surface influences the variation of the $y$-component of the velocity. The thermal plume generated by the heating unit rises in the $y$-direction and is likely to separate from the surface that is $6 \mathrm{~mm}$ away from the heated surface close to $y=100 \mathrm{~mm}$, and spread to the interior of the cavity where a circulation is formed. Similarly, the departing location on the surface $12 \mathrm{~mm}$ away from the heated surface could be $y=200 \mathrm{~mm}$. This is in good agreement with previous studies in [41][42].

Fig.10. $y$-component of the velocity $(v)$ measured by TIV and PIV in a Line1, $\mathbf{b}$ Line2, $\mathbf{c}$ Line3 and $\mathbf{d}$ the representative lines.

With the exception of minor differences, the results of PIV6 and TIV for the three lines are similar in terms of the general trends exhibited. For heights within the range $0-200 \mathrm{~mm}$, the differences between TIV and PIV6 are relatively greater, specifically at the corresponding locations of Line 2 . In Line 2, the TIV results within the range $y=0-200 \mathrm{~mm}$ are specifically affected by the abovementioned shadows. Compared to other locations, the temperature fluctuation gradient is significant, and the shadows interfere with the acquisition of temperature fluctuation data. Overall, the TIV result are similar to those of the PIV6.

The airflow in Line 2 is close to free flow, but the airflow in Lines 1 and 3 is unavoidably 
disturbed by the sidewalls. Similarly, the flow in the location of $y=0-200 \mathrm{~mm}$ is also perturbed by the heating unit, resulting in the actual flow in Lines 1 and 3 differing from that in Line 2. Overall, the distributions of the vertical velocity profiles in Line 1 and Line 3 are similar. However, the vertical profiles in Line 1 are not similar to Line 3, although all the geometric boundary conditions are similar. For actual flow, the boundary conditions on both sides cannot be fully controlled and kept consistent. In fact, the flow that should theoretically be symmetrical is asymmetrical, with a number of inconsistencies. Some studies [12][43] have shown that the asymmetric flow exists under symmetrical boundary conditions. The TIV and PIV measurements has indicated that the results of TIV and PIV6 are apparently closer within the range $y=200-1000 \mathrm{~mm}$. There appears to be a linear relationship between the results of TIV and PIV in the range $y=0-200 \mathrm{~mm}$. A linear fit is applied for Line 1 within the range, as shown as Table 1, and the results yield good linear relationships with high R-square values. In addition, it can be concluded that the results of TIV and PIV6 are almost identical.

Table 1. Linear fit equations of TIV, PIV6 and PIV12 in line 1

The $x$-component of the velocity $(u)$ in Line1 is plotted in Fig. 11. Unlike the y-component $(v)$, the $x$-component of the velocity $(u)$ in the vertical direction is significantly smaller in magnitude and is close to zero. No significant pattern exists for the $x$-component of the velocity.

Fig.11. $x$-component of the velocity in line 1

In order to further investigate the relationship between the PIV6 and TIV, scatter plots and linear fit plots of the $y$-components of the velocities evaluated by TIV and PIV6 in the three lines are shown in Fig. 12 and Fig. 13. These values are positively correlated with high R-square values and fit well on the line $y=a x$ with high correlation coefficients, thereby indicating that there is a linear relation between TIV and PIV6.

Fig.12. Relationship between $y$-component of the velocity $(v)$ measured by TIV and PIV6.

Fig.13. Relationship between velocity (V) measured by TIV and PIV6.

\subsection{Uncertainty analysis of PIV}

The uncertainty analysis of PIV can be discussed from two aspects: the system error and the statistical error [44]. The system errors are caused primarily by tracing particle' following 
performance, image deformation, and random particle displacement in the image analysis [44]. The motions of the tracer particles suspended in the air are affected by numerous factors, including air velocity, tracer particle density, and gravity. The tracer particle following performance is defined according to the ratio of the tracer particle velocity with the fluid velocity. In this study, the amplitude ratio of the tracer particles and the air is used to evaluate the following performance. The detailed calculation method can be referred to [12][45]. In addition, the amplitude ratio is 0.99 and the error contributed by following performance can be neglected. Typically, the error caused by image deformation is close to $0.3 \%$ [44]. As stated in [46], the random displacement error during the experiment was approximately 0.07 pixels, that will produce a relative error up to $4 \%$ for the corresponding mainstream region $(>0.1 \mathrm{~m} / \mathrm{s})$. In conclusion, the maximum system error is approximately $4.3 \%$.

The statistical error of PIV is caused primarily by random sampling errors. The PIV random sampling error can be evaluated according to the central limit theorem, assuming that the random sampling errors are normally distributed [47][48]. In this case, the random sampling error of the time-averaged velocity can be estimated:

$$
S(A)=\frac{1}{\sqrt{k}} \cdot z_{\alpha / 2} \cdot \frac{a^{\prime}}{A}
$$

where $S(A)$ is the random sampling error of the time-averaged velocity, $k$ is the sampling size, $\frac{a^{\prime}}{A}$ is the ratio of the root mean square velocity and the time-averaged velocity. $z_{\alpha / 2}$ is a coefficient determined by the confidence interval. When the confidence interval is $95 \%$, the value of $z_{\alpha / 2}$ is 1.96. Fig. 14 shows the random sampling errors when the time-averaged velocity is $0.1 \mathrm{~m} / \mathrm{s}$. The random sampling errors would increase with decreasing time-averaged velocity. In this regard, when the time-averaged velocity is smaller than $0.1 \mathrm{~m} / \mathrm{s}$, the random sampling errors would be greater, and vice versa. However, it is not reasonable to evaluate the errors of the entire measurement regions with the errors of regions with small velocities. It is necessary to choose a modest velocity to calculate the error. As can be seen from Fig.7.(a), in the bulk of regions, the velocities are greater than $0.1 \mathrm{~m} / \mathrm{s}$, and only a small percentage of the regions have insignificant velocities and are approximately stationary. Therefore, the random sampling error is calculated when the time- 
averaged velocity is $0.1 \mathrm{~m} / \mathrm{s}$. According to Fig. 14, it is possible to obtain a relatively high accuracy with 300 images pairs. In this case, the random sampling error is $6.1 \%$. Therefore, the uncertainty of the PIV measurement is approximately $10.4 \%$.

Fig. 14. Random sampling error of the time-averaged velocity

\section{Discussion}

\section{Considering the investigation in this study, a number of limitations and error sources that could} influence the velocity measurement via the use of thermal image series should be carefully monitored in the measurements.

Quantifying the uncertainty related to the field of view of the camera is critical. The camera needs to be positioned to ensure that the field of view encompasses the target measurement domain and avoids inclusion of other surfaces as much as possible. In addition, the camera lens should be perpendicular to the measured surface as even a slight tilt could cause image distortions. In addition, the camera performance is critical for acquiring high-quality thermal images and it should be accurately calibrated prior to the measurements.

The background noise could provide meaningless information, adding work to the acquiring of thermal spot displacements. Shadows from other surfaces on the transparent target surface and the surface local heterogeneities could obscure the thermal features and interfere with the acquisition of the time-series thermal images. In addition, longwave reflections from adjacent surfaces will increase the measurement error, specifically when radiation energy emitted by the target surface is very small. According to the Stefan-Boltzmann law, the radiation emitted by the target object depends primarily on the surface emissivity and the temperature. Typically, the surface temperature is fixed for the selected measurement object. The surface emissivity should be sufficient to increase the radiation energy measured by the thermal infrared camera.

The abovementioned preprocessing of the captured images can decrease the influence of the local surface heterogeneities and low surface emissivity. Additional experiments were conducted to analyze the influence of local heterogeneities, surface emissivity, and the pre-processing of the captured images on the measurement accuracy. The area of the electric heating unit was $500 \mathrm{~mm} \times$ $400 \mathrm{~mm}$ and it was installed in the same location as in the previous experiment. The surface 
temperature of the heating unit was maintained at $40{ }^{\circ} \mathrm{C}$. The experiments were conducted under three working conditions. Case 1 was used as the control group and has no change with the wall surface. The surface emissivity input to the thermal infrared camera is 0.90 , that is the emissivity of the Plexi-glass. In Case 2, the wall surface was pasted with four pieces of black tape to add surface heterogeneities, as shown in Fg.15 (b). In Case 3, the wall surface was pasted with silver tape to alter the surface emissivity, and the surface emissivity was 0.83, as shown in Fig.15 (c). Except for the target surface differences, the other boundary conditions remained the same. A region with dimensions of $500 \mathrm{~mm} \times 445 \mathrm{~mm}$ above the heating unit was measured by TIV. The velocity profiles in the red line in Fig. 15 are discussed. Fig. 16 shows the velocity profiles of the three cases and the gray shaded areas in Fig.16 (a) are the areas where the black tape was pasted. It can be concluded that the existence of local heterogeneities and low emissivity, in Fig.16 (a) and (b) respectively, do influence the accuracy of the velocity measurements. However, through pre-processing of the captured images, the deviation can be improved to some extent.

Fig. 15. Schematic diagram of wall surfaces of the three conditions: a Casel, the unaltered surface and surface emissivity is 0.90 , b Case 2, the surface with pieces of black tape and surface emissivity is $0.90, \mathbf{c}$ Case 3 , the whole surface with silver tape and surface emissivity is 0.83 .

Fig. 16. Velocity profiles of the additional experiment to show the influence of local heterogeneities, surface emissivity and the pre-processing of the captured images on the measurement accuracy

\section{Conclusion and outlook}

This study proposed a method for measuring the two-dimensional distribution of airflow near a heated surface by tracking surface temperature images. The near wall airflow under natural convection near the heated surface in the cavity was investigated by TIV and PIV. By comparing the PIV results, the feasibility of TIV in the measurement of indoor airflow field near heated surfaces under natural convection was discussed. The primary conclusions obtained from this study are as follows.

(1) A velocity visualization method referred to as thermal image velocimetry is proposed. This method obtains the velocity field by acquiring the temperature fluctuations near the heated surface, and the algorithm is similar to the algorithm used for PIV. In this method, the only 
instrument is a thermal camera that is portable and easy to operate compared to the PIV method. It is noteworthy that this method can obtain the velocity field without access to the flow field. Thermal image velocimetry has an advantage over the traditional measurement techniques and

(2) Based on the analysis of the time sequence of thermal images, the basic characteristics of the has good application prospects in predicting the airflow field, such as the microclimate near the terminal heating units in buildings.

flow field near the heated surface were evaluated. The spatial variability of the velocity appeared to comprise three patterns in the vertical direction: (1) a high velocity region, (2) a moderate velocity, and (3) a low velocity and relatively lagging region. The fluid velocity increased in the vicinity of the heating unit and attained a maximum value in the range $y=0$ 200. The velocities of the three vertical lines exhibited linear relationships with high $R$-square values at locations within the range $200-1000 \mathrm{~mm}(y=200-1000 \mathrm{~mm})$.

(3) The summarized results indicated that the indoor velocity field near a heated surface can be accurately evaluated using thermal image time series. Comparison of the velocities indicated that the velocity fields obtained by thermal image velocimetry and the one measured by particle image velocimetry at the surface that was $6 \mathrm{~mm}$ away from the heated surface was similar. Specifically, it was observed that there was good agreement between the PIV measurements and the velocity deduced from the thermal images. In the representative lines, they were positively correlated with high R-square values. There was a linear relationship between TIV and PIV6.

Although the initial investigations yielded encouraging results, additional studies could be conducted to improve the reliability of TIV in indoor air velocity measurements. In this study, the wavelength of the PIV laser was inconsistent with the sensitive wavelength of the infrared camera. If both measurements were conducted at the same time, the laser of PIV would inevitably affect the acquisition of surface temperature fluctuations in TIV measurement. In result, the two measurements were performed separately. Although the boundary conditions maintained good consistency, the turbulent nature of the flow makes the velocity of the air variable locally and temporally. Future studies can carried out using TIV in conjunction with another reliable velocity measurement method for synchronous measurement. From the experimental comparison in this 

study, it could be seen that in the prediction of the flow field near the heating surface, the TIV and 508 PIV results exhibited a high degree of consistency. This indicates that the TIV method has the 509 potential for independently measuring indoor airflow fields. Specifically. When it comes to actual 510 full-scale airflow measurements near the actual heating terminal units, as mentioned above, PIV 511 could be unsuitable. However, as an alternative experimental method, TIV requires only a thermal 512 infrared camera and, therefore, has greater operability. Regarding the application of TIV in the velocity measurement of actual full-scale room, further studies are required. 


\section{References}

[1] Y. Sun, Y. Zhang, An Overview of room air motion measurement: technology and application, HVAC\&R Res. 13 (2007) 929-950.

[2] S.C. Li, D.F. Huang, N. Meng, L.F. Chen, L.H. Hu, Smoke spread velocity along a corridor induced by an adjacent compartment fire with outdoor wind, Appl. Therm. Eng. 111 (2017) 420-430.

[3] F. Wang, C. Liang, X. Zhang, Experimental study on frost suppression for ASHP combining superhydrophobic heat exchanger and air flow, Appl. Therm. Eng. 136 (2018) 666-673.

[4] J. Yan, X. Lu, Q. Wang, Y. Kang, J. Li, J. Zhou, Y. Zhang, Z. Lv, S. Sun, Experimental and numerical study on air flow uniformity in the isobaric windbox of a $600 \mathrm{MW}$ supercritical CFB boiler, Appl. Therm. Eng. 122 (2017) 311-321.

[5] M. Caciolo, P. Stabat, D. Marchio, Full scale experimental study of single-sided ventilation: Analysis of stack and wind effects, Energy Build. 43 (2011) 1765-1773.

[6] S. Kumar, R.B. Grover, H. Yadav, P.K. Vijayan, U. Kannan, A. Agrawal, Experimental and numerical investigation on suppression of thermal stratification in a water-pool: PIV measurements and CFD simulations, Appl. Therm. Eng. 138 (2018) 686-704.

[7] G. Cao, H. Awbi, R. Yao, Y. Fan, K. Sirén, R. Kosonen, J. (Jensen) Zhang, A review of the performance of different ventilation and airflow distribution systems in buildings, Build. Environ. 73 (2014) 171-186.

[8] D.H. Shin, D.K. Sohn, H.S. Ko, Analysis of thermal flow around heat sink with ionic wind for highpower LED, Appl. Therm. Eng. 143 (2018) 376-384.

[9] G. V. Kuznetsov, M. V. Piskunov, R.S. Volkov, P.A. Strizhak, Unsteady temperature fields of evaporating water droplets exposed to conductive, convective and radiative heating, Appl. Therm. Eng. 131 (2018) 340-355.

[10] S. Fu, P.H. Biwole, C. Mathis, Particle tracking velocimetry for indoor airflow field: A review, Build. Environ. 87 (2015) 34-44.

[11] A. Sattari, Particle image velocimetry visualization and measurement of airflow over a wallmounted radiator, Int. J. Vent. 14 (2015) 289-302.

[12] X. Zhang, G. Su, J. Yu, Z. Yao, F. He, PIV measurement and simulation of turbulent thermal free convection over a small heat source in a large enclosed cavity, Build. Environ. 90 (2015) 105-113.

[13] G. Hetsroni, T.A. Kowalewski, B. Hu, A. Mosyak, Tracking of coherent thermal structures on a heated wall by means of infrared thermography, Exp. Fluids. 30 (2001) 286-294.

[14] T.A. Kowalewski, A. Mosyak, G.Hetsroni, Tracking of coherent thermal structures on a heated wall, Exp. Fluids,34(2003).

[15] X. Cao, J. Liu, N. Jiang, Q. Chen, Particle image velocimetry measurement of indoor airflow field: A review of the technologies and applications, Energy Build. 69 (2014) 367-380.

[16] S. Kacmaz, E. Ercelebi, S. Zengin, S. Cindoruk, The use of infrared thermal imaging in the diagnosis of deep vein thrombosis, Infrared Phys. Technol. 86 (2017) 120-129.

[17] H. Wang, Z. Zou, Z. Shi, B. Li, Detecting ship targets in spaceborne infrared image based on modeling radiation anomalies, Infrared Phys. Technol. 85 (2017) 141-146.

[18] S. Bejannin, P. van Beek, T. Stieglitz, M. Souhaut, J. Tamborski, Combining airborne thermal infrared images and radium isotopes to study submarine groundwater discharge along the French Mediterranean coastline, J. Hydrol. Reg. Stud. 13 (2017) 72-90.

[19] Q. Tang, J. Liu, J. Dai, Z. Yu, Theoretical and experimental study on thermal barrier coating (TBC) uneven thickness detection using pulsed infrared thermography technology, Appl. Therm. Eng. 114 (2017) 770-775.

[20] D. Soler, P.X. Aristimuño, M. Saez-de-Buruaga, A. Garay, P.J. Arrazola, New calibration method to measure rake face temperature of the tool during dry orthogonal cutting using thermography, Appl. Therm. Eng. 137 (2018) 74-82.

[21] T. Astarita, G. Cardone, G.M. Carlomagno, C. Meola, A survey on infrared thermography for convective heat transfer measurements, Opt. Laser Technol. 32 (2000) 593-610.

[22] S.S. Halkarni, A. Sridharan, S. V. Prabhu, Measurement of local wall heat transfer coefficient in randomly packed beds of uniform sized spheres using infrared thermography (IR) and water as working medium, Appl. Therm. Eng. 126 (2017) 358-378.

[23] A. Christen, J. Voogt, Linking atmospheric turbulence and surface temperature fluctuations in a street canyon, in: Proc 7th international conference on urban climate, Yokohama, Japan, 2009.

[24] R Voogt, Visualisation of turbulent exchange using a thermal camera, in: Proc 18th Symposium on Boundary Layers and Turbulence Conference, Stockholm, Sweden, 2008.

[25] A. Christen, A. Garai, A. Inagaki, J. Kleissl, F. Meier, D. Scherer, R. Vogt, J. Voogt, Mapping coherent structures responsible for heat exchange between land-surfaces and atmosphere using 
time-sequential thermography, in: Coherent Flow Structures II Conference, Vancouver, Canada, 2011.

[26] A. Garai, E. Pardyjak, G.J. Steeneveld, J. Kleissl, Surface Temperature and Surface-Layer Turbulence in a Convective Boundary Layer, Boundary-Layer Meteorol. 148 (2013) 51-72.

[27] M. Katurji, P. Zawar-reza, Forward-Looking Infrared Cameras for Micrometeorological Applications within Vineyards, Sensors. 16 (2016) 2-11.

[28] G. Anriban, Interaction between surface and atmosphere in a convective boundary layer, University of California San Diego, California, USA, 2013.

[29] A. Garai, J. Kleissl, Air and Surface Temperature Coupling in the Convective Atmospheric Boundary Layer, J. Atmos. Sci. 68 (2011) 2945-2954.

[30] A.M. Grudzielanek, J. Cermak, Capturing Cold-Air Flow Using Thermal Imaging, Boundary-Layer Meteorol. 157 (2015) 321-332.

[31] A. Inagaki, M. Kanda, S. Onomura, H. Kumemura, Thermal Image Velocimetry, Boundary-Layer Meteorol. 149 (2013) 1-18.

[32] Y. Fan, Y. Li, J. Hang, K. Wang, Diurnal change of wall flows and energy balance on the south facing wall when background wind is absent, in: Proc 10th Pacific Symposium on Flow Visualization and Image Processing Conference, Naples, Italy, 2015.

[33] A. Tiddens, K. Risthaus, M. Röger, H. Stadler, B. Hoffschmidt, Induced Infrared Thermography: Flow visualizations under the extreme conditions of an open volumetric receiver of a solar tower, Int. J. Heat Fluid Flow. 65 (2017) 105-113.

[34] X. Sun, Y. Li, Review of the development of temperature measurement technology with infrared thermal imager, Laser \& Infrared. 38 (2008) 101-104.

[35] Y. Li, X. Sun, Research on Measurement Temperature Technology and Application of Infrared Thermal Imaging, Mod. Electron. Tech. 32 (2008) 112-115.

[36] R. C. Gonzalez, R. E. Woods, Digital Image Processing, Prentice Hall, 2002.

[37] G. Leclerc, S.W.M. Fast fourier transforms: a tutorial review and a state of the art, Signal Processing. 19 (1990)259-299.

[38] J. P. Lewis, Fast Normalized Cross-Correlation, Circuits, Syst. Signal Process. 28 (2009) 819-843.

[39] J. Nogueira, A. Lecuona, P.A. Rodríguez, Data validation, false vectors correction and derived magnitudes calculation on PIV data, Meas. Sci. Technol. Meas. Sci. Technol. 8 (1997) 1493-1501.

[40] P. Wang, Research on the new method of post-processing of PIV, Dalian university of technology, Dalian, China, 2004.

[41] K. Ben. Nasr, R. Chouikh, C. Kerkeni, A. Guizani, Numerical study of the natural convection in cavity heated from the lower corner and cooled from the ceiling, Appl. Therm. Eng. 26 (2006) 772775.

[42] M. Corcione, Effects of the thermal boundary conditions at the sidewalls upon natural convection in rectangular enclosures heated from below and cooled from above, Int. J. Therm. Sci. 42 (2003) 199-208.

[43] A. Li, C. Yang, T. Ren, X. Bao, E. Qin, R. Gao, PIV experiment and evaluation of air flow performance of swirl diffuser mounted on the floor, Energy Build. 156 (2017) 58-69.

[44] X. D. Cao, Experimental study of the airflow characteristics in a passenger aircraft cabin mockup with 2D-PIV, Tianjin university, Tianjin, China, 2015.

[45] A. T. Hjelmfelt, L. F. Mockros, Motion of discrete particles in a turbulent fluid, Int. J. Sci. Res. 16(1996):149-161.

[46] M. Raffel, C. Willert, S. Wereley, J. Kompehans, Particle image velocimetry: a paractical guide $2^{\text {nd }}$ edition, Springer, 2007.

[47] H. W. Coleman, W. G. Steel, Experimental, validation, and uncertainty analysis for engineers, John Wiley \& Sons, 2009.

[48] J. Stafford, E, Walsh, V. Egan, A statistical analysis for time-averaged turbulent and fluctuating flow field using particle image velocimetry, Flow Meas. Instrum. 26 (2012) 1-9. 
Figures

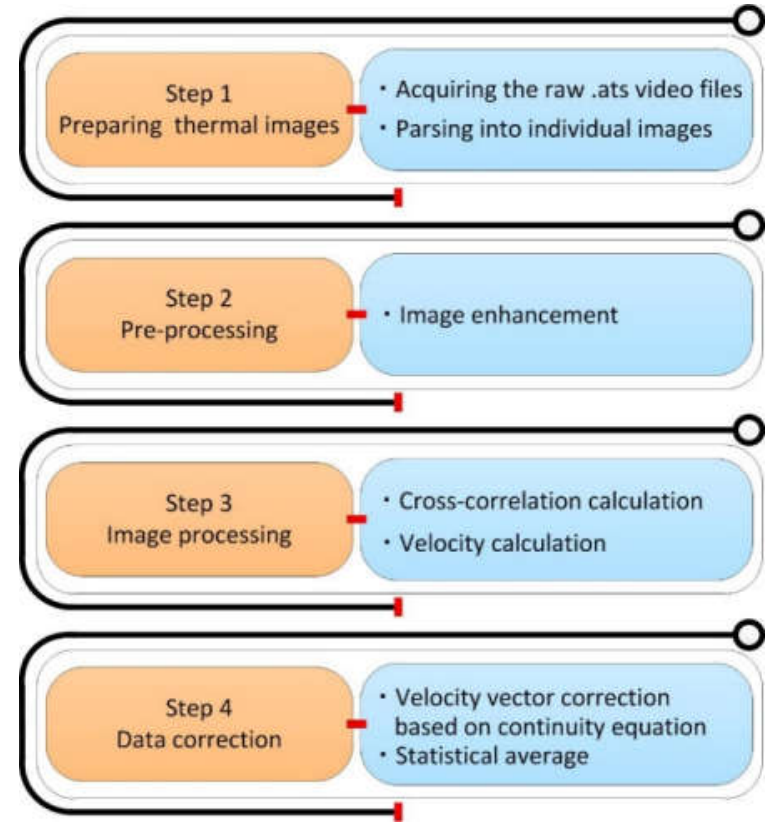

Fig. 1. Technical approach 


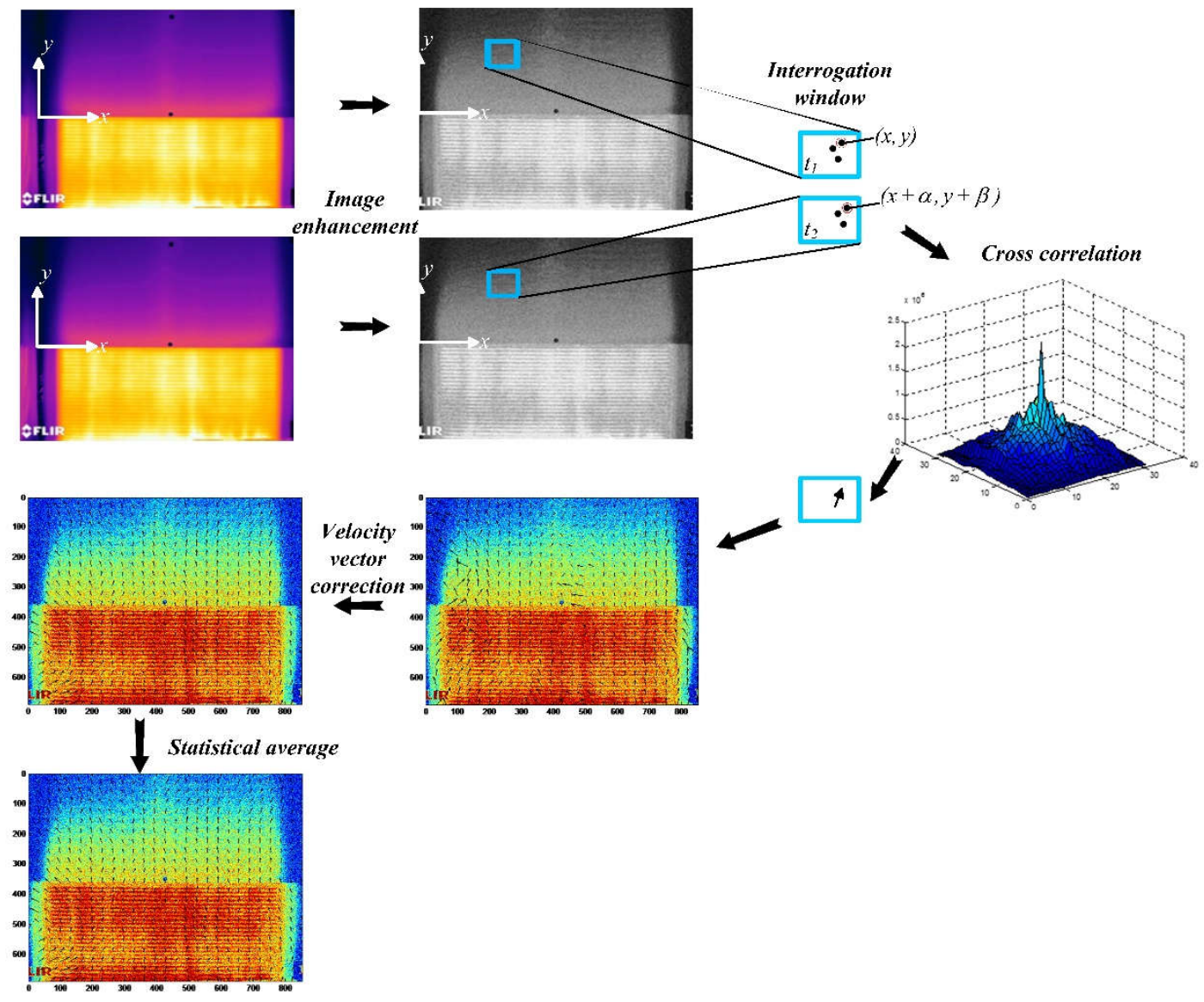

Fig. 2. The data processing for TIV measurement 


\begin{tabular}{|l|l|l|}
\hline$(-1,1)$ & $(0,1)$ & $(1,1)$ \\
\hline$(-1,0)$ & $(0,0)$ & $(1,0)$ \\
\hline$(-1,-1)$ & $(0,-1)$ & $(1,-1)$ \\
\hline
\end{tabular}

Fig. 3. The sketch for velocity grid in the step of data correction 


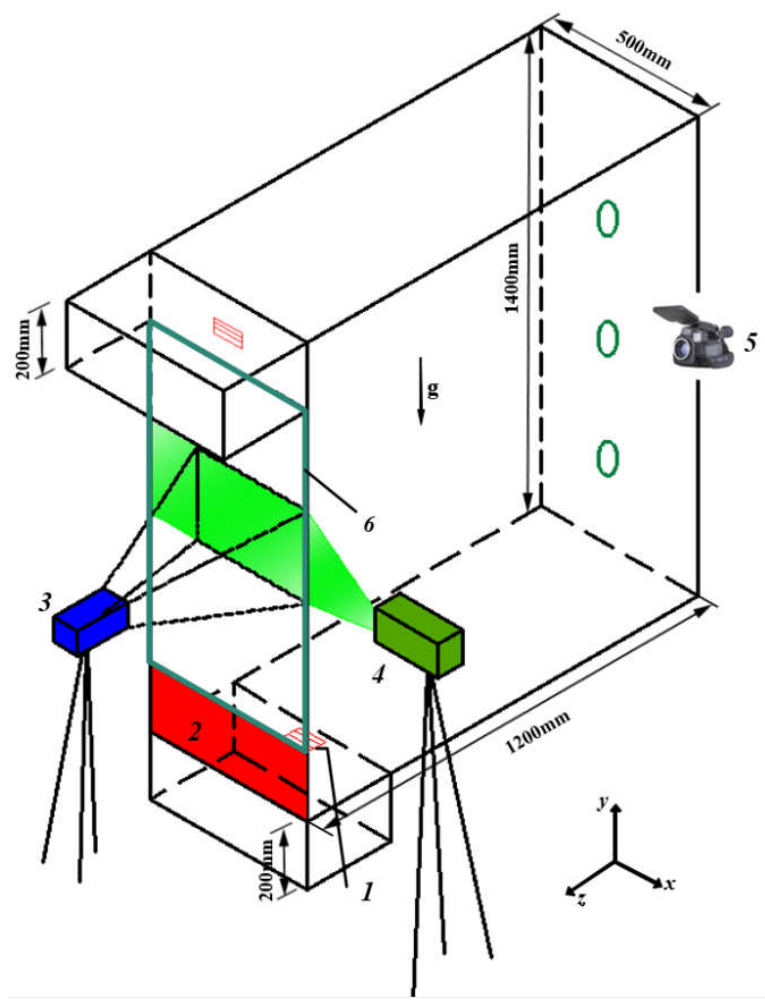

Fig. 4. Experimental setup: 1. Tracer particle inlet 2. Heating unit 3. CCD camera 4. Laser 5. Infrared camera 6. Visualized surface 


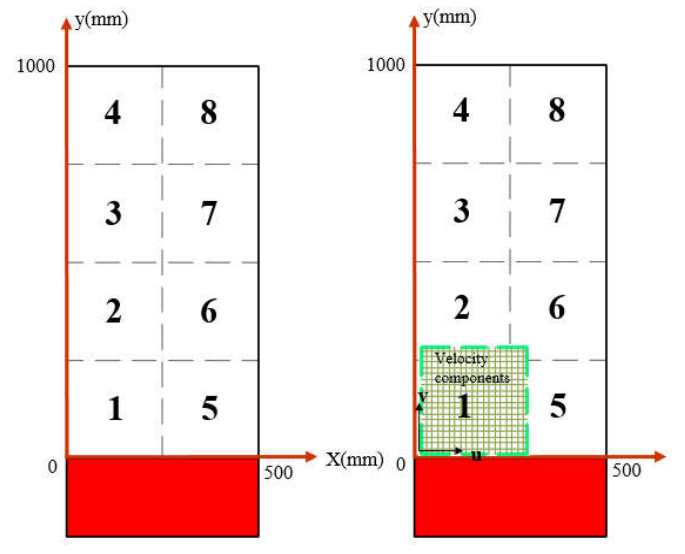

(a)

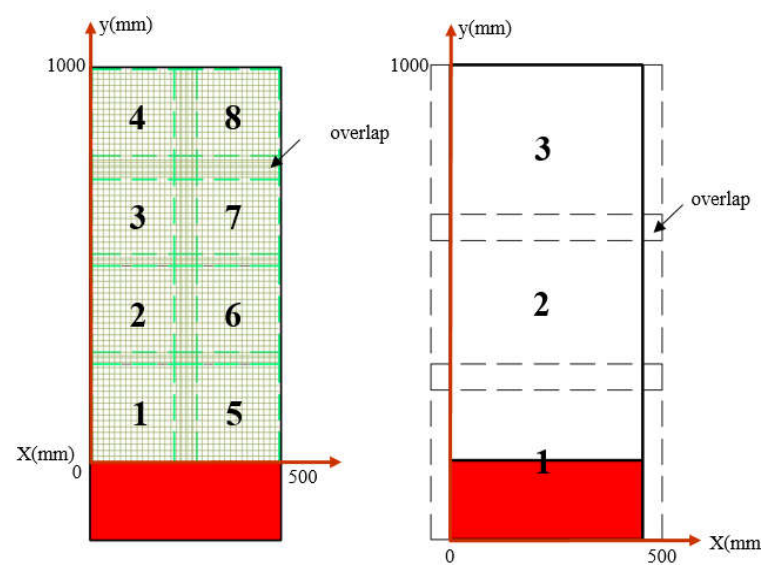

(b)

Fig. 5. Schematic of measurement domains a. PIV b. TIV 


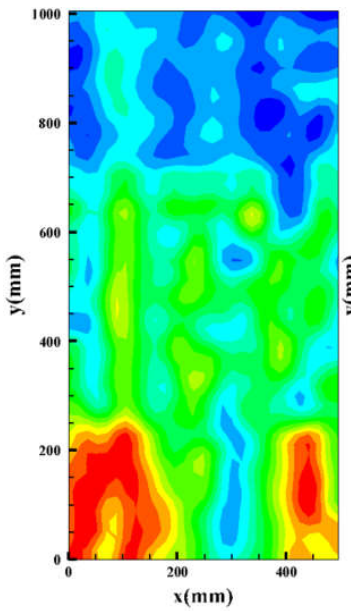

(a)

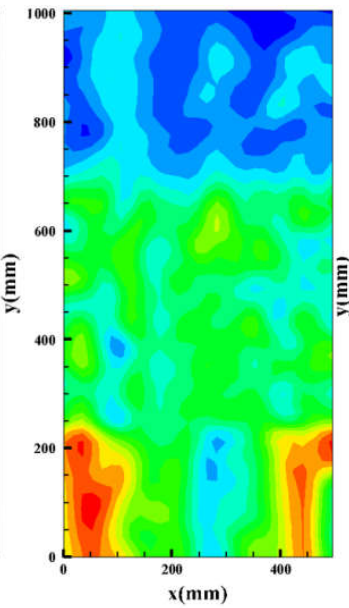

(b)

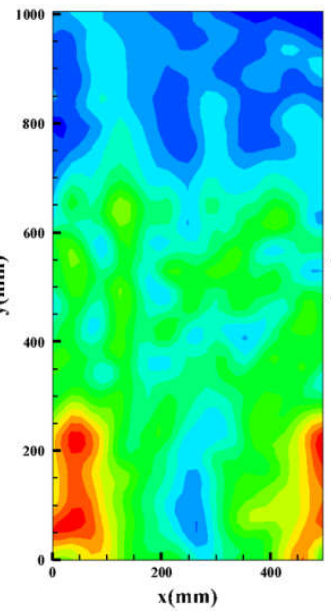

(c)

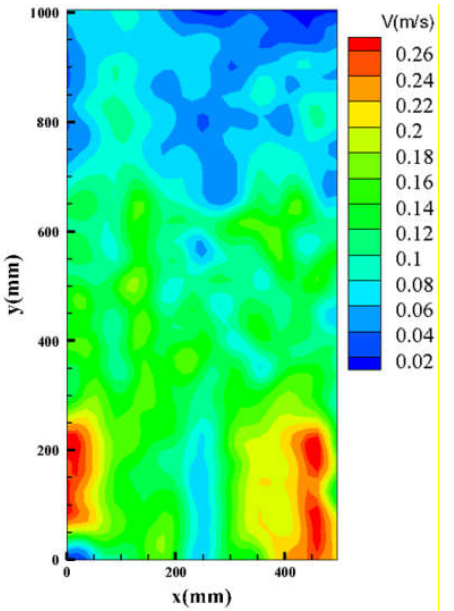

(d)

Fig. 6. Instantaneous velocity measured by TIV, with (a) to (d) representing four consecutive moments. 

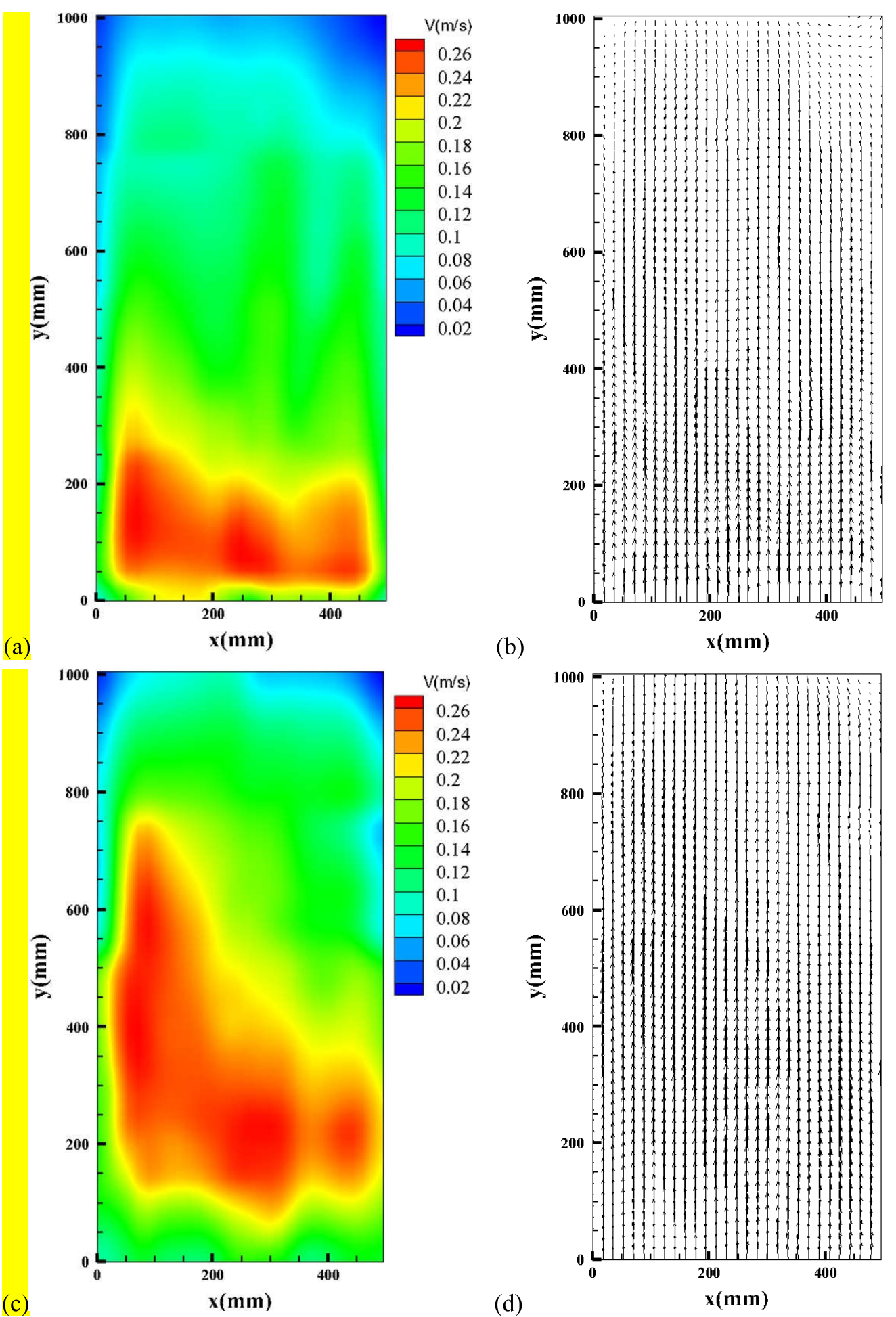

(b)

$\mathbf{x}(\mathbf{m m})$

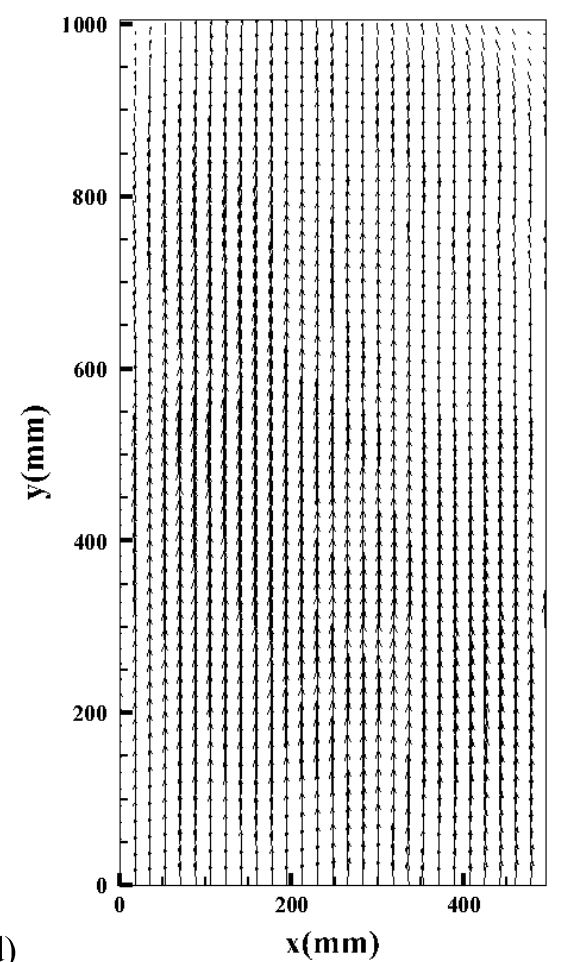

Fig. 7. Velocity distributions near the heated surface evaluated by PIV: a (b), c (d) velocity contour

(velocity vector) of the planes $6 \mathrm{~mm}$ and $12 \mathrm{~mm}$ away from the heated surface. 

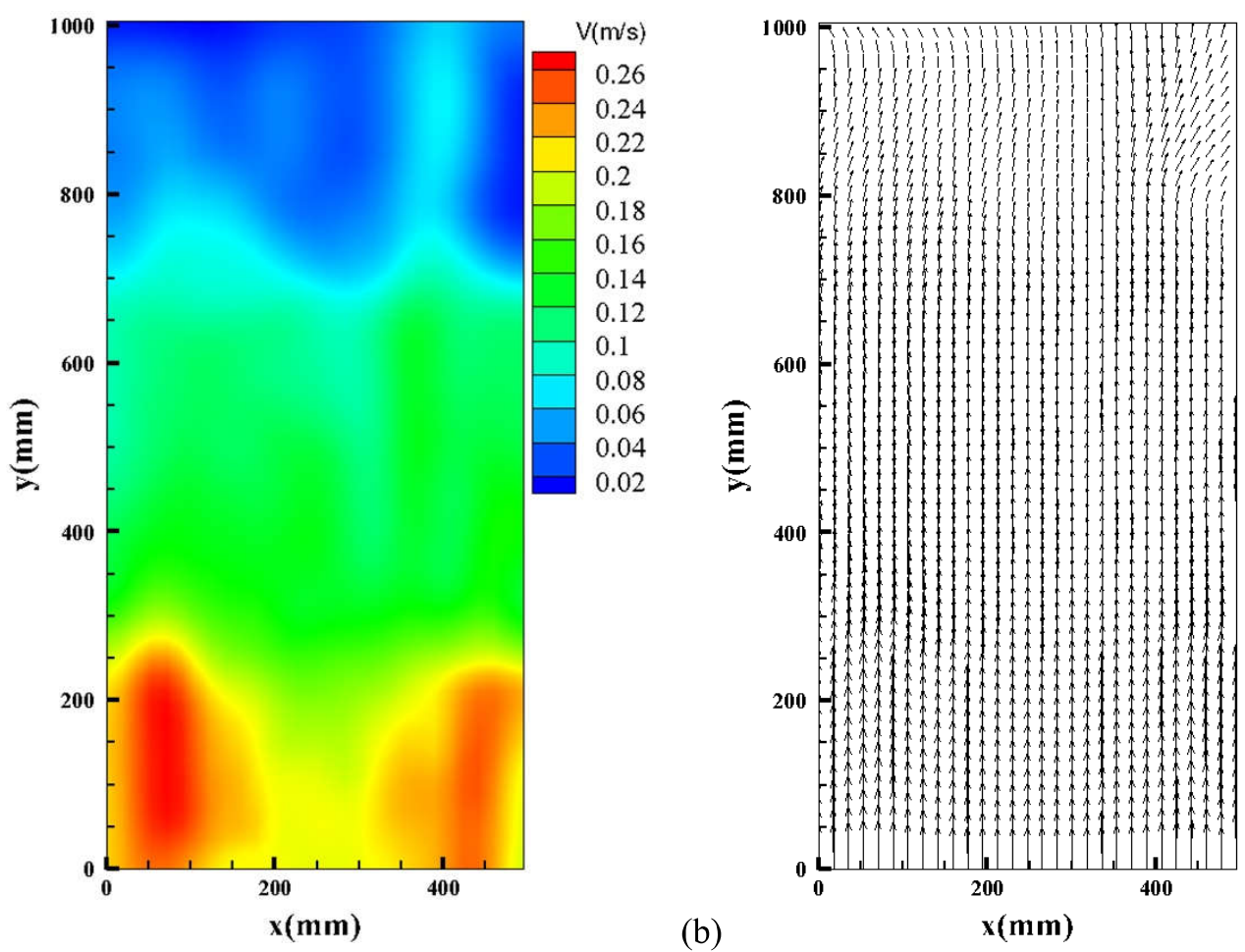

Fig. 8. Velocity distributions near the heated surface evaluated by TIV: a velocity contour, $\mathbf{b}$ velocity vector. 


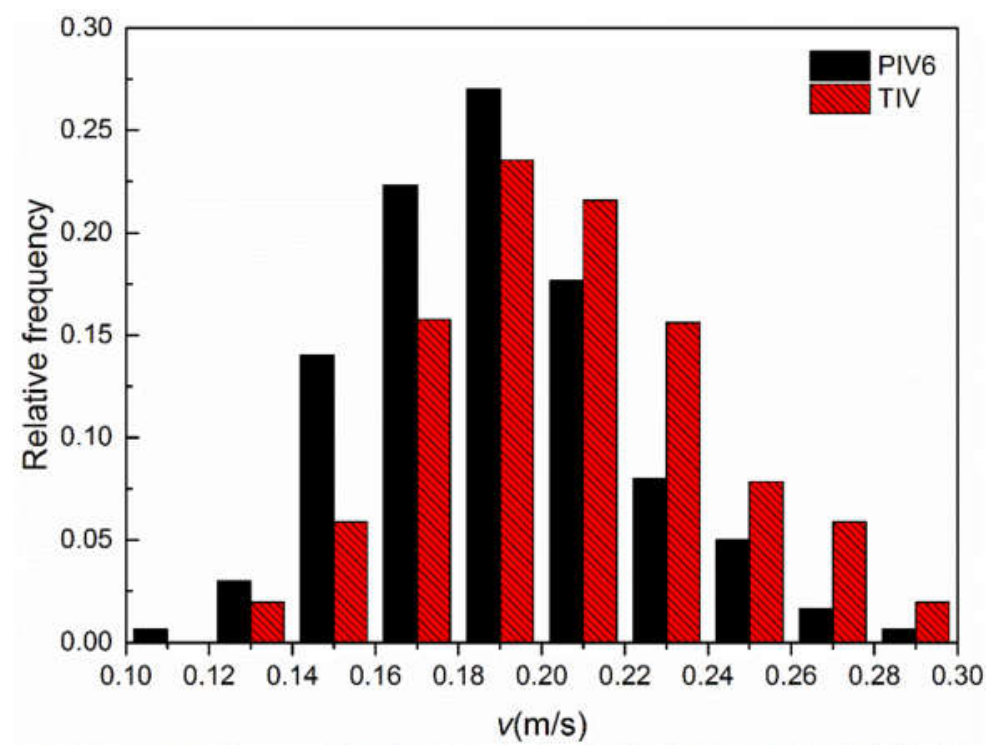

Fig. 9. Histogram of $y$-component of the velocity $(v)$ evaluated by TIV and PIV6. 


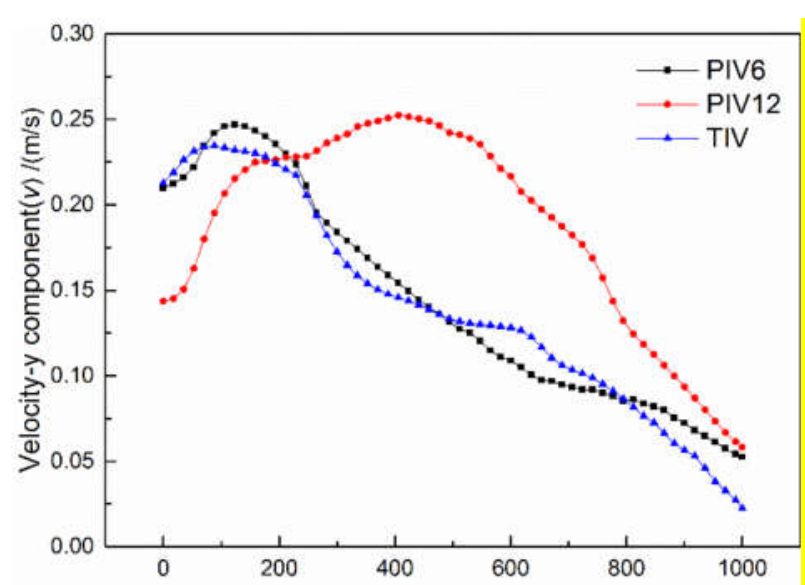

(a)

Height(y)/mm

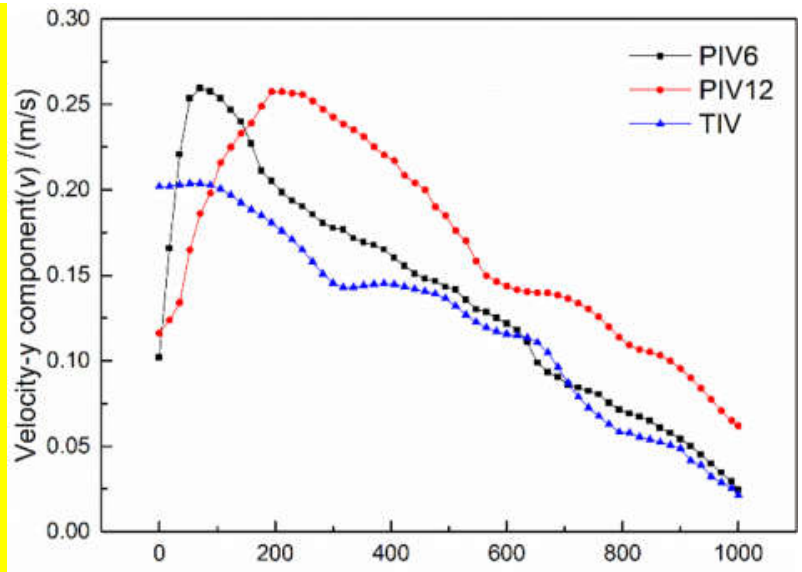

(b)

Height(y)/mm

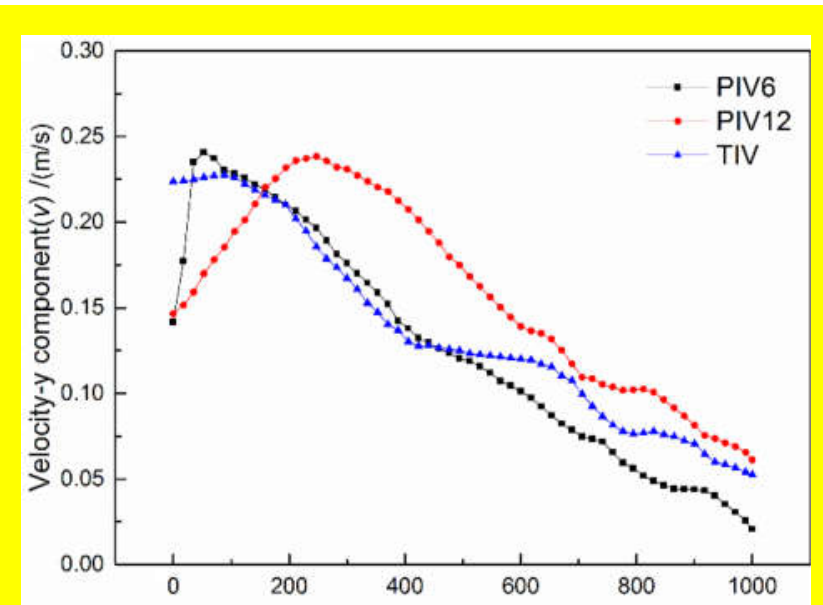

(c)

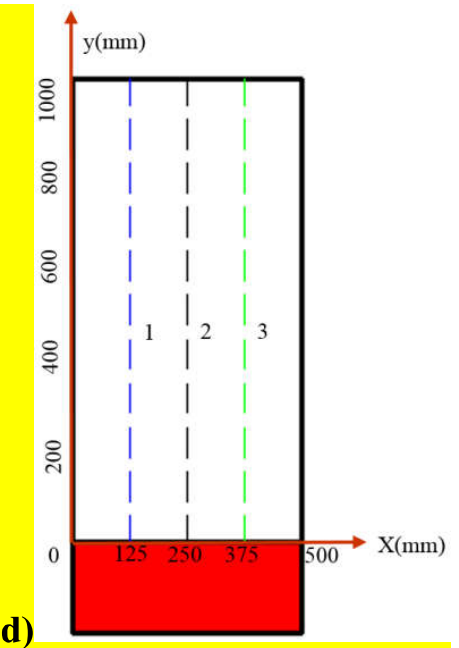

Fig. 10. $y$-component of the velocity $(v)$ measured by TIV and PIV in a Line1, $\mathbf{b}$ Line2, $\mathbf{c}$ Line 3 and $\mathbf{d}$ the representative lines. 


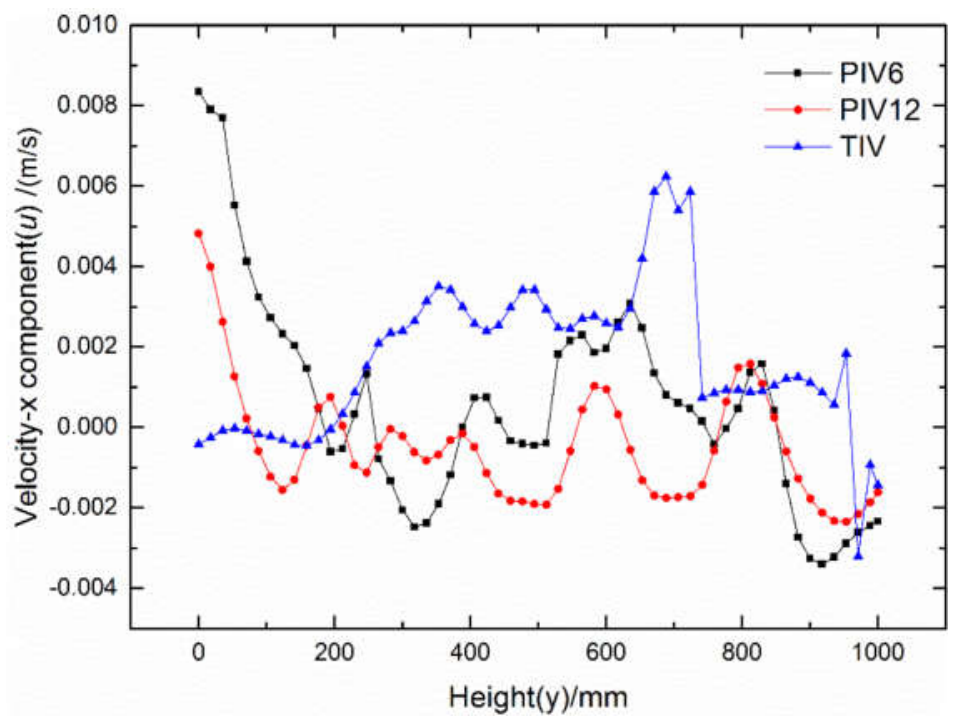

Fig. 11. $x$-component of the velocity in line 1 


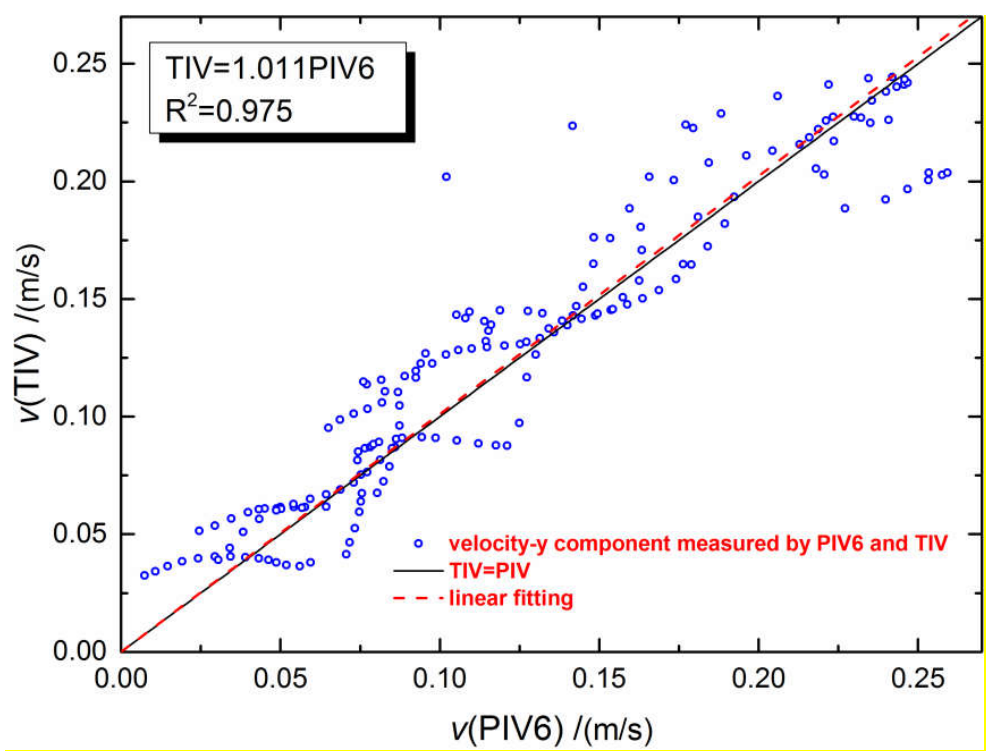

Fig. 12. Relationship between $y$-component of the velocity $(v)$ measured by TIV and PIV6. 


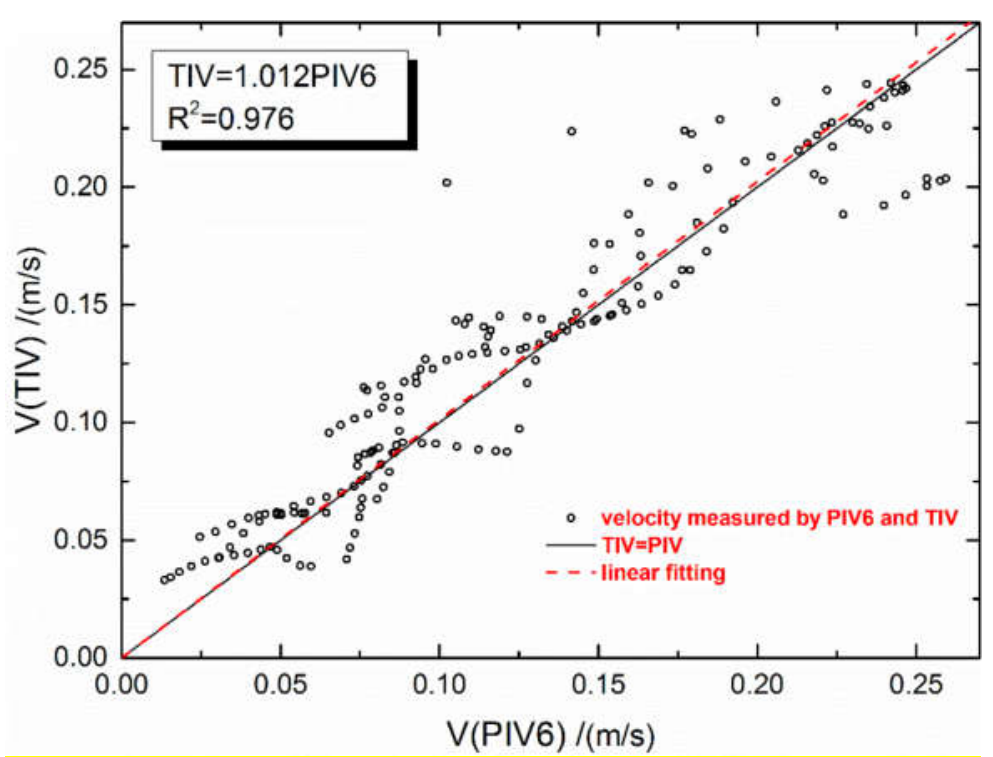

Fig. 13. Relationship between velocity (V) measured by TIV and PIV6. 


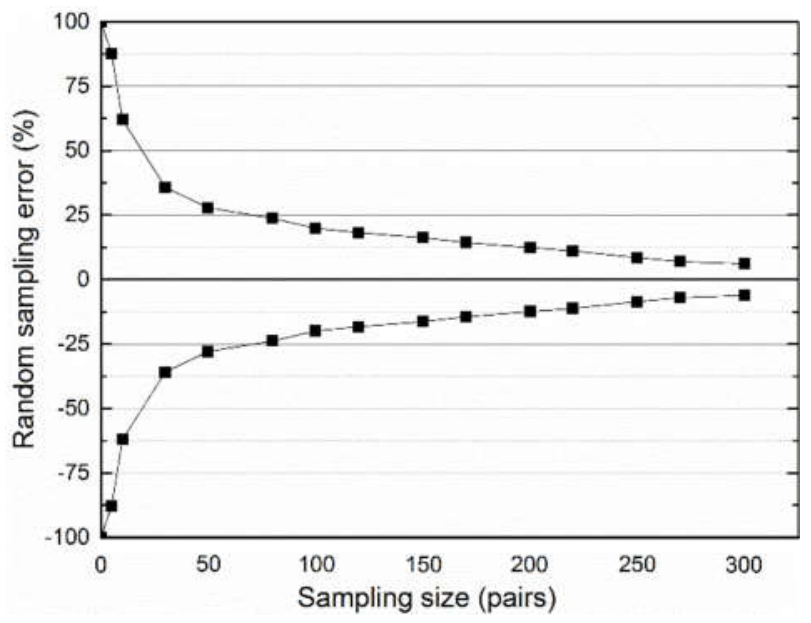

Fig. 14. Random sampling error of the time averaged velocity 


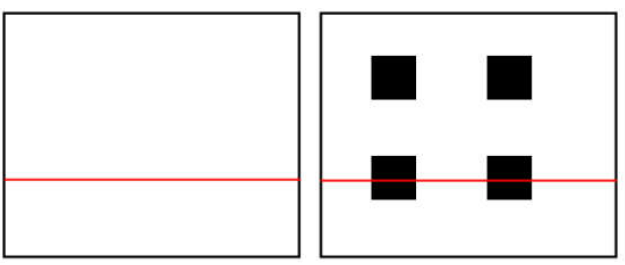

(a)

(b)

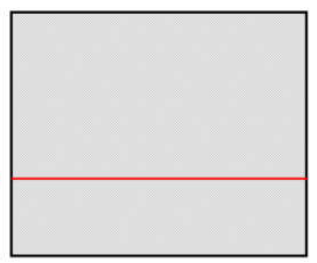

(c)

514 Fig. 15. Schematic diagram of wall surfaces of the three conditions: a Case1, the unaltered surface and

515 surface emissivity is $0.90, \mathbf{b}$ Case 2 , the surface with pieces of black tape and surface emissivity is 0.90 , c Case 3 , the whole surface with silver tape and surface emissivity is 0.83 . 


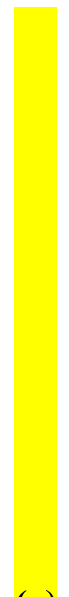

(a)

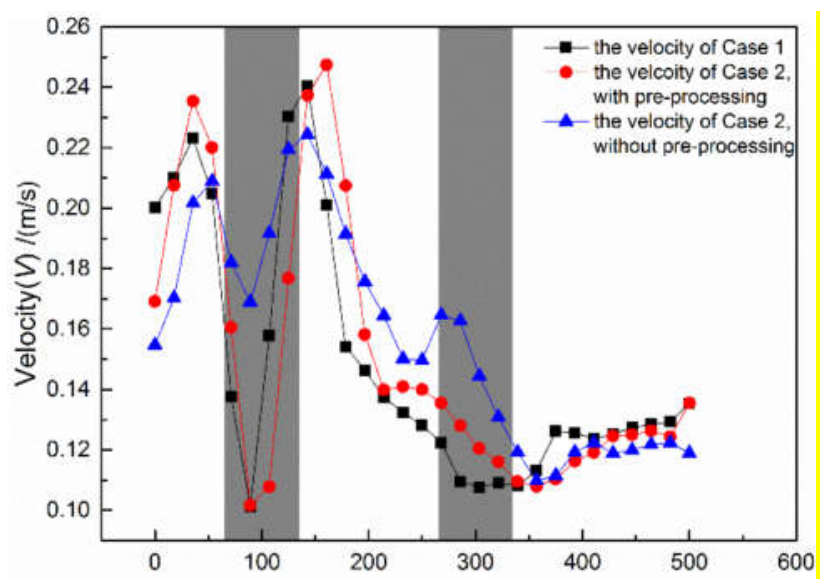

Position $(\mathrm{x}) / \mathrm{mm}$

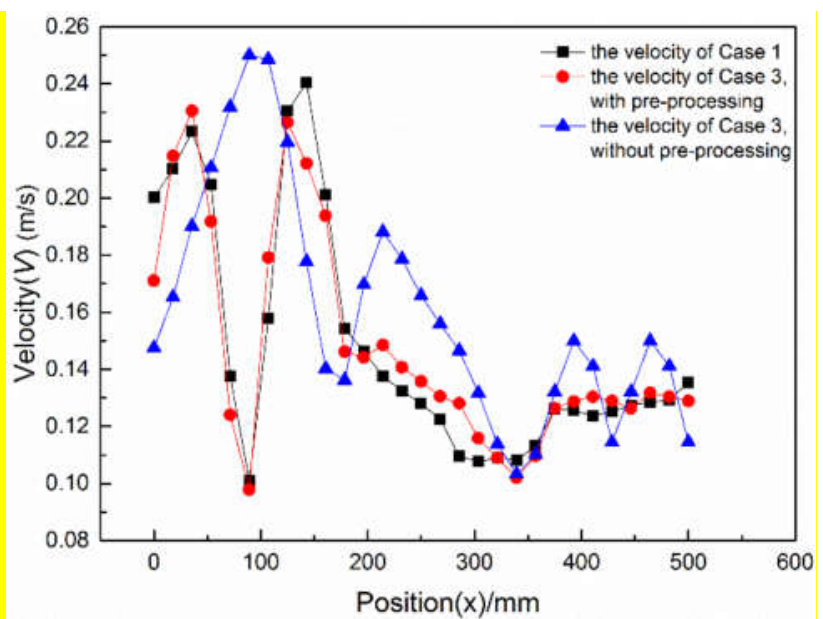

(b)

Fig. 16. Velocity profiles of the additional experiment to show the influence of local heterogeneities, surface emissivity and the pre-processing of the captured images on the measurement accuracy 


\section{Table}

Table 1. Linear fit equations of TIV, PIV6 and PIV12 in line 1

\begin{tabular}{ccc}
\hline Measurement & Linear regression equations & $R^{2}$ \\
\hline TIV & $v=0.218-1.89(y / 10000)$ & 0.962 \\
PIV6 & $v=0.246-2.169(y / 10000)$ & 0.995 \\
PIV12 & $v=0.311-2.495(y / 10000)$ & 0.981 \\
\hline
\end{tabular}

